\title{
'My [asexuality] is playing hell with my dating life': Romantic identified asexuals negotiate the dating game
}

Tiina Vares

\begin{abstract}
While academic attention to asexuality has increased in recent years, there is still relatively little research into the relational lives of romantic identified asexuals, and less still on the gendered dimensions of these. This article aims to address these research gaps by examining the ways in which 13 self-identified romantic asexuals living in New Zealand experience and navigate dating practices/finding somebody. I employ a feminist poststructuralist approach to explore the ways in which the pervasiveness of hook up culture and gendered discourses of sexuality both constrain and enable possibilities for developing partnered relationships with non-asexuals.
\end{abstract}

\section{Keywords}

Asexuality, dating, gender, relationships, sexuality

\section{Introduction}

Human asexuality has increasingly become the focus of popular and academic attention in the past fifteen years. The establishment of the online Asexual Visibility and Education Network (AVEN) in 2001 and Anthony Bogaert's (2004) article 'Asexuality: Prevalence and Associated Factors in a National Probability Sample' heralded an upsurge of interest in the topic. David Jay, the founder of AVEN, has become somewhat of a celebrity featuring on television talk shows, podcasts, magazine and newspaper articles, and the recent documentary (A)sexual (2011) directed by Angela Tucker. Academic scholarship was initially slow to engage with asexuality although this has started to change in the past few years (for example: Dawson et al., 2016; MacNeela and Murphy, 2015; Robbins et al., 2016).

AVEN's definition of an asexual person as 'someone who does not experience sexual attraction' has, Mark Carrigan argues, gained widespread assent while also concealing a great deal of diversity (2011: 476). Within the asexual community there is considerable diversity of identities, with one list on AVEN including over fifty identifications. One of the primary differences is between those who identify as romantic and as aromantic and based on the presence or absence of romantic attraction. While neither experience sexual attraction, romantic individuals experience romantic attraction and often desire an intimate, non-sexual relationship. However, for individuals who identify as grey-asexual or demi-sexual, sexual attraction is a possibility in special circumstances, for example, once a relationship has developed (Carrigan, 2011: 470). Thus while lack of sexual attraction is a common feature of asexuality, it is not a universally shared definition (Scherrer, 2008: 626). It is also important to 
acknowledge the fluidity of asexual identities. Carrigan (2011) indicates that sexual fluidity emerged in his study and it was also discussed by the participants in the study discussed in this article (for example, moving across the identifications of aromatic, romantic, demiasexual etc.)

The burgeoning scholarship on asexuality has increasingly focused on the diversity of asexual identities and, in particular, the subjective dimensions of asexual experience (Scherrer, 2008; Carrigan, 2011; Robbins et al., 2016). With respect to making asexual identities culturally intelligible this focus, Jacinthe Flores argues, is crucial for 'making sense of how diverse bodies are experienced and understood as asexual' (2014: 73). However, it was only 2 years ago that Ela Przybylo and Danielle Cooper indicated that research on asexuality was 'almost entirely absent in queer, feminist and critical sexuality studies' (2014: 298). Since then some work which is informed by feminist and queer theorising has emerged, for example, Karli Cerankowski and Megan Milks (2014) Asexualities, Feminist and Queer Perspectives, although it is still rather minimal.

Another area of asexual experience which remains under-researched is that of romantic relationships, even though some researchers have highlighted the prevalence of these among asexuals (Dawson et al., 2016). Some recent work suggests that asexual individuals experience difficulties when wanting to engage in a partner relationship (Haeffner, 2011; Van Houdenhove et al. 2015). This includes forming relationships and negotiating what constitutes intimacy and non-asexual partners' sexual expectations. Matt Dawson et al.'s study on how asexual people negotiate the boundaries of intimacy considers the different ways in which asexuals engage in 'sex as a practice of intimacy' and how some of their participants feel 'excluded from areas of intimate life or practices of intimacy' (2016: 357). With respect to the former, engaging in sexual activity can result from societal expectations and pressure, the desire to 'pass' as sexual, or 'gifting' sex for the good of the relationship (Dawson et al., 2016).

Dawson et al., employ a symbolic interactionist approach and argue that 'rather than seeking to transform the nature of intimate relationships, asexual people make pragmatic adjustments and engage in negotiations to achieve the forms of physical and emotional intimacy they seek' (2016: 350). In this article, and more explicitly in Scott and Dawson (2015), the authors critique what they refer to as 'political research on asexuality [which] has been conducted predominantly within the poststructuralist tradition' (2015: 7). They argue that 'this body of political research' evident in the work of Pryzbylo (2011) and others, 'treats asexual intimate lives as harbingers or radical change' when the 'focus should be on exploring the intersection of intimate practices with other life circumstances (such as age and class background) and other sexual identities' (italics added) (Dawson et al., 2016: 362). This is because of a lack of 'empirical evidence' in poststructuralist research on asexuality, their reading/critique of poststructuralism as focused on discourse rather than lived experience, 
and the 'individualistic' nature of poststructuralist theorizing with respect to marginalising 'social encounters, relationships and interaction' (Dawson et al., 2016: 352).

My aim here is not to debate the differences in these theoretical approaches, nor deny disciplinary and theoretical positions, but to pick up on Ela Pryzbylo's suggestion that there are consistent features in asexual research in 'an otherwise diverse interdisciplinary field' (2013: 193) and that attention to these could potentially contribute to asexuality studies, that is, asexuality as an interdisciplinary method. As a sociologist, feminist and poststructuralist, my own research attends to the lived experiences of participants, their perspectives and their social relationships/interactions, as does that of Dawson et al. My analysis, like theirs, considers they ways in participants' practices 'open up potential relationship forms' and also close down future potentialities (Dawson et al., 2016: 362). While being aware our different epistemological positions, I suggest that my paper addresses a research gap in asexuality research (in particular, empirical feminist poststructural research on the dating practices of romantic asexuals) and also builds on consistencies in/across existing asexuality research on intimacy and relationships, rather than cross-disciplinary critique. My hope is that it encourages us to think more about the interdisciplinarity of approaches to asexuality (Przybylo, 2013: 193).

This article reflects on a study with 15 self-identified asexuals living in New Zealand. The focus is primarily on the experiences and perspectives of the 13 romantic identified asexuals, in particular, their practices of dating or trying to 'find somebody'. I explore they ways in which they participate in/navigate the 'dating game' with non-asexuals, the gendered challenges they face and negotiate, and the opportunities and limitations of 'finding somebody' in BDSM (Bondage and Discipline, Domination and Submission, and Sadism and Masochism) communities.

\section{Methodology}

Recruitment of self-identified asexuals living in New Zealand took many months (March to August 2016). While the aim of the study was to talk to 20 people only 16 responded to recruitment posts and 15 agreed to participate. A number of online sites were used, for example, AVEN, a queer youth support group, a student recruitment website and Asexuals New Zealand Facebook. Ten of the participants responded from the latter. Although previous studies have recruited primarily through AVEN, for this project only one participant responded to the AVEN posts.

The participants were self-identified asexuals, living in New Zealand and between 18 and 60 years of age. There was a noticeable divide between those in their late teens/early twenties (18-23 years old) and those in their late forties and older (47-60 years old). Previous studies have indicated concerns about reaching older asexuals through their use of online 
recruitment strategies. I was able to recruit three participants aged 47 years and over through snowballing, with another two from online posts. Fourteen participants lived in New Zealand's major cities (Auckland, Hamilton, Wellington, New Plymouth, Rotorua, Christchurch, Dunedin) and one was from a South Island town. With respect to ethnicity, the majority were Pakeha, one was Māori ${ }^{1}$ and one Iranian. Interviews were conducted either in person (10) or by phone (5). Some participants asked for a phone interview and for others, my travelling to their location was not practical at the time. The interviews lasted between 35 and 120 minutes, with the phone interviews usually being the shortest (with the exception of one which was the longest).

The analysis of the interviews in informed by a feminist poststructuralist approach that, drawing on the work of Foucault (1990[1978]), understands subjectivity or identity as constructed in discourse (see Gavey, 1989, 2005; Weedon, 1987). This approach acknowledges the dual nature of subject positioning - participants are both positioned by and position themselves in relation to various discourses. I employ a form of thematic discourse analysis that identifies themes in the transcripts and sees language as constitutive of meaning and meanings as social (Braun and Clarke, 2006). A substantial thematic category I identified in the talk of participants was the difficulties of 'finding somebody' and negotiating the heterosexual 'dating game' (most romantic identified participants identified as heteroromantic). Within this, key themes included the prevalence of a hook up culture, the often gendered responses participants received from potential or actual non-asexual dates, participants' often gendered responses to dates, and the use of BDSM communities to try to 'find somebody'. These themes frame the analysis in the following sections.

When presenting extracts from interviews I have omitted word repetitions and all speech hesitations (i.e., all terms such as ' $u m^{\prime}$ ' and 'ah'). The presence of three consecutive dots [...] indicates a portion of speech has been cut, pseudonyms are used for all participants, and the number following the pseudonym indicates the participant's age.

\section{Romantic asexuality: 'It's quite hard to find somebody'}

'The problem is being romantic because it means that you want to be with somebody and it's quite hard to find somebody'. (Sarah)

Of the 13 participants who identified as romantic (including grey-romantic), ten were heteroromantic, two were bi-romantic and one identified as 'simply asexual' although she desired a partnered relationship with a man or woman in which there was no 'sex'. For most of these participants 'sex' included a range of sexual practices such as oral sex, touching the genitalia and breasts, in contrast to the dominant heteronormative discourse in which 'sex' is equated with penile-vaginal penetration. All 13 romantic identified participants articulated a range of issues with finding and/or negotiating a relationship in which there was no sex. An ideal 
relationship was usually described as one with another asexual, however, the possibility of meeting another asexual in person (let alone someone to connect with) was seen as unlikely ${ }^{2}$. This is due to a number of factors including: the relatively small number of asexuals ${ }^{3}$ in New Zealand (given its population of 4.5 million); the online location of the local and international asexual community; the small number of social and/or support groups (primarily in the larger cites); the often private nature of asexual identity ${ }^{4}$; and the relatively poorly run asexual dating websites. Only one participant, Hallie, spoke of a relationship with other asexuals she met through AVEN - these were online relationships because of physical distance. So while the ideal relationship was imagined as one with another asexual, this was seen as a long shot at best. For those wanting a romantic relationship, non-asexuals were thus seen to offer more opportunities for partnered/romantic relationships.

With the exception of Angela (aged 56 years), none of the participants were in relationships at the time of the interviews (although Kathy, aged 60 years, was recently widowed). Several participants expressed a strong desire to be in a relationship, for example, Sarah, who identifies as bi-romantic, had been in a number of relationships (some with sex prior to identifying as asexual) and continued to actively seek a monogamous relationship, with nonsexual touch, that would work for her. However, several others who desired a relationship were no longer seeking one because of the difficulties of finding somebody. While Mike had been 'constantly looking for a romantic relationship' and 'wanting a serious partner [since he was] thirteen', he indicated that he was no longer 'intentionally dating'. Kaitlyn had also given up on the 'dating game':

Kaitlyn (27): Like it gets quite lonely, if you know what I mean, and [...] I've been on Tinder and things like that but it's difficult to be in the dating game and try and be in a relationship when [...] you want to find a partner and not have sex with them because everybody, I just feel like everybody, is out for it, pretty much.

Although Gabriella did 'occasional spurts' on dating sites, like Kaitlyn, she found that 'people wanted to do physical things straight away'. The notion that 'everybody' is out for sex can be understood in the context of what Pryzbylo refers to as our sexusociety- which acknowledges the central roles sexuality and sex play in our society and in our lives (2011: 446). Integral to sexusociety is the prevalence of a hook up culture in which people get together for a sexual encounter with no promise or desire to develop a relationship. Although this is not a new phenomenon, some suggest it has replaced dating as the dominant way for young people to get together (see Bogle, 2007 and 2008: Garcia et al., 2012). Social media and dating apps, in particular, offer a range of ways to meet a wider pool people and/or hook up. Several participants talked about using dating websites, particularly Tinder and OK Cupid. Although some of the participants had used asexual dating sites, none had any success with them. Many 
participants thus focused on non-asexual dating sites in their search for a date or potential relationship.

One issue participants grapple with is when to tell one's potential or actual date that 'sex will not happen here' (Kaitlyn). Some participants used the direct approach - Sarah's online dating profile states, 'Asexual ahoy, touch yes: sex no'. However, this has not deterred approaches from those interested in hook ups, for as Sarah laughingly explains, they 'see yes and sex together and ignore the colon'. She hasn't found dating sites very fruitful and was using BDSM (Bondage and Discipline, Domination and Submission, and Sadism and Masochism) websites at the time of the study (see below). Kaitlyn, in contrast to Sarah, argues that disclosing one's asexuality can terminate the date, 'if you tell a date, "oh, I'm asexual", they'll go "I don't want a bar of that"'. Most of the asexual participants in Carol Haefner's (2011) study also indicated that disclosing their asexuality meant the end of a potential relationship. This presents a double bind for asexuals: an early disclosure can discourage contact or end a date, yet waiting until one gets to know one's date before disclosing does not necessarily prevent the same outcome.

\section{Negotiating gendered responses to (romantic) asexuality}

Disclosure of asexuality to a potential or actual date presents further challenges, in particular, the possibility of getting negative and/or abusive comments. Gabriella received distressing comments on online dating sites which included 'being treated like a lab specimen' and 'invalidated'. Such comments, together with the hook up culture of the sites, have prompted her to give up on using them. Respectful comments, she suggests, would make a considerable difference:

Gabriella (32): If I knew that I would find someone that would be completely okay with my identity and would negotiate with me respectfully [...] then I would probably seek out relationships.

A lack of public awareness about asexuality, combined with the pervasive discourse that all humans have the capacity to have erotic experiences and response, informs reactions to asexuality such as: it doesn't exist; it's just a phase; you'll grow out of it; and you just haven't found the right person yet. Although such responses have been discussed by others (see Robbins et al., 2016; MacNeela and Murphy, 2015) there has been little consideration of the ways in which these are potentially gendered. In this study the female participants were more likely to be told that they had not 'found the right man yet', and the male participants - that it was 'just a phase' or that they were just 'fooling themselves' as asexuality wasn't a 'real thing'. Mike articulated this gendered dynamic in relation to the different challenges he sees asexual men and women facing: 
Mike (22): Well for a guy being asexual - people assume that it doesn't exist, whereas a girl being asexual people just assume that she's just that way because she hasn't had the right guy yet. And it's like, people assume different things and because of that we face separate challenges.

The discursive construction of feminine sexuality as passive and requiring male 'sexpertise' to 'awaken' continues to circulate in popular culture (for example, John Gray's 'Men are from Mars, Women are from Venus' argument). Not having found the right man can be read as a way of articulating this construction - the 'right' man is the one who will 'awaken' the unresponsive sexuality of his female partner. Offered as a way of explaining asexual women's lack of sexual attraction, it reproduces the discourse of female sexual passivity and simultaneously denies the legitimacy of the asexual female subject. There are different ways in which this construction of feminine sexuality can be employed. While not having found the right man is not explicit with respect to sexuality, the idea that 'good sex' (presumably penile-vaginal penetration) is what a woman needs is far from ambiguous:

Gabriella (32): [...] a lot of people come at you going, 'I'm going to fix you, my sex is so good that you will want it'.

Tiina: Have you had that?

Gabriella: I've had that from men on dating sites mostly, so that's why I avoid them like the plague now [laughs].

No men in my study or in the literature I've reviewed to date, spoke of responses from prospective female dates which offered to 'fix them' through 'good sex'. This rests not only on the construction of feminine sexuality as passive but also the construction of masculine sexuality as natural, driven, oriented around penile performance or, as Bernie Zilbergeld puts it, men are always interested in and always ready for sex (cited in Plummer 2005: 185). The centrality of (hetero)sexuality to dominant masculinity has been well documented (see Gavey 2005; Hollway 1984, 1989): a real man performs in sex, sex is centred on a hard penis and what's done with it, and sex equals intercourse (Zilbergeld cited in Plummer 2005). The men Gabriella encountered positioned themselves as embodiments of this construction with their penile performance framed as fixing her sexual disinterest and transforming her into a sexually desiring subject.

For asexual men, the decoupling of masculinity from sexuality challenges this fundamental tenet of masculinity. This was articulated by both male and female participants when they suggested that it is harder for men to identify as asexual:

Olivia (18): I think for guys, it's a little harder to be asexual because they're expected to have such a high sex drive as well. 
Kaitlyn (27): [...] it might be a lot more weird and frowned upon for [men] to not want sex.

Kaitlyn and Olivia both draw on the male sex drive discourse (Hollway 1984, 1989) to support their claim that asexual men are more likely to receive negative responses because they do not embody this discourse. As indicated above, they are also more likely to be seen as 'weird' and/or encounter 'disbelief' (Mike). Mike talks about how others simply do not accept the possibility of male asexuality, 'they'll say something like "you've got to be joking" or "you're a guy"'. The unspoken question here is, how can you be a guy and not be interested in sex? Philip (one of the two participants in the study who identified as aromantic) also talked of the difficulty of being a man who had never had sex or been in a relationship:

Philip (49): Because when I was a teenager and when I was in my 20s, I should've been having lots of sex or this very powerful urge should've been coursing through me and I should've been behaving in a certain way and I wasn't and that was a real problem for some people who, you know, thought I should be doing this. [...] In my 30s [...] it was more of an issue for me that I'd never had a relationship. I'd never had sex, never had a relationship. This was very embarrassing for me as a male, never to have had sex because, of course males are supposed to go out and do the girls and ra ra ra, proving your masculinity, yeah. Whoa. So even though I was happy to tell people I wasn't interested in a relationship, I wasn't very happy saying, well you know, if they asked more, I would have to admit I'd never had a relationship. [...] Now I'm a 49 year old bald guy. Who cares if he's not having any sex? He's not supposed to have sex. Just thinking about him having sex is disgusting [laughs]. So it's got a hell of a lot easier as I got older, yeah.

Philip similarly orients himself to the male sex drive discourse to describe how a man should be in his youth. His experience of not being able to take up this dominant masculine subject position of strongly desiring and 'do[ing] the girls' became more of an issue as he got older. He found it relatively easy to tell others he wasn't interested in a relationship as this implied he'd had one before and was between relationships. This slant enabled him to be seen as a 'normal' heterosexual man. To admit he' $d$ never had a relationship/sex dispelled this illusion of heteronormalcy and was more problematic. However, age has made a difference to how he is read/understood. Philip indicates that no-one expects him - as a 49 year old bald guy to be sexually active and that it is thus 'easier' to be an older asexual man. This is informed by the construction of those in later life as not sexually desirable, not sexually desirous and not sexually capable 5 .

For the two romantic identified men in the study, their desire to be in a relationship meant that they had to confront expectations of male sexual desire/performance with potential 
dates/partners more directly. Mike (who defines himself as 'sex repelled') had dated a number of girls and women - some prior to identifying as asexual (at age 16), while Aidan (who describes himself as 'repulsed by sex') only dated prior to identifying as asexual at age 20. For both men the expectation and/or pressure to have penile-vaginal penetrative sex was difficult to manage and they attempted it even though this was not what they wanted. Aidan's girlfriend broke up with him after they 'kind of did it together, like had sex [...] I mean, it couldn't really happen'. He also mentioned breaking up with a woman because 'she was so sexual'. Similarly, Mike did 'try [sex] a couple of times early on, but it just didn't work out', and he also ended a couple of relationships because of the pressure to have sex 6 , 'It's not worth hanging onto a relationship if I have to do that'.

For Aidan, identifying as asexual was a significant 'kind of like a coming out moment' and he has not been interested in dating since then. In contrast, Mike's desire and search for a serious relationship continued for a while. He did 'tell people [he was asexual] beforehand' and although 'things are good to start out with normally l'll end up getting cheated on quite quickly or they dump me early'. He suggests they 'wound up breaking it off with me because they wanted quote unquote more' (that is, sex). It is significant that Mike disclosed his asexuality from the outset and that this did not seem to deter women at first. However, the short-duration of these potential relationships due to an expectation of sex suggests (as Mike indicates) that women did not believe that asexuality could be a 'real thing' for a man. Mike summed up his situation with the comment that his asexuality 'is playing hell with my dating life'. He is no longer dating because he has had 'just one horrible romantic life' although he still desires a partner (he describes himself as 'super huggy').

Given dominant constructions of masculine sexuality and the pervasiveness of hook up culture, it seems likely that young non-asexual women struggle to make sense of asexual men. However, in spite of his 'horrible romantic life' neither Mike nor Aidan saw themselves as being 'less of a man', as did a few of the participants in Padraig MacNeela and Aisling Murphy's (2015: 807) survey. Rather they, like many of the male participants in MacNeela and Murphy's study, represented themselves as disrupting dominant discourses of masculine sexuality. Aidan, for example, talked about being seen 'as not manly' but that current 'standards of manly' are not what he wants. Although his 'asexuality is taking away things' he also appreciates that 'asexuality stops me from being that hegemonic masculine [man]'.

Female participants also talked about dating situations in which male partners wanted 'more'. However, unlike male participants, they positioned themselves as 'leading' their male dates 'on'. This discursive positioning involves two aspects: concern for their date (being lead on) and feeling responsible and/or guilty themselves (for leading on). It is possible Aidan and Mike experienced such feelings however these were not articulated in the interview context. Therefore, my focus is on a potential gendered dimension in the talk of the participants with respect to employing the notion of 'leading on'. In her survey of asexuals, Heffner also notes 
that some of her participants wrote about leading prospective partners on. She does not however reflect on this as potentially gendered although the two examples she uses are from women (2011: 99).

Madison was one of the female participants who employed the notion of leading or stringing along a date:

Madison (23): I dated a few people throughout high school, I have since as well. I [...] enjoy dating [...]. But I always find, after five or six dates, the guy is [...] obviously wanting more and [...] I feel awful because I feel like I've strung them along.

Madison goes on to say she does not date much anymore because, 'I feel like I'm leading them on if it gets too far, so I don't like putting myself and them in that position'. So like Mike and Aidan, Madison has given up on dating because dates generally want 'more'. A 'guy obviously wanting more' can be located within a male sexual drive discourse in which men are positioned as always interested in sex and once sexually aroused needing to be satisfied by orgasm. Within the terms of this discourse, the woman is positioned as the object that precipitates men's natural sexual urges (Hollway, 1984) and it isn't right or fair for her to stop before his orgasm. If she does stop, she is framed as a 'tease' or as 'leading him on'. With the privileging of masculine sexuality, the woman is at fault in this situation (clearly this also operates for non-asexual women). To be a 'tease' is not a desirable subject position - hence Madison 'feeling awful' and responsible for putting her dates and herself in this position. Kaitlyn takes this a step further when she reflects on a hypothetical dating situation:

Kaitlyn (27): I would feel that way if I went on a date with someone, I would feel like I would have to eventually have sex with this person because that's the expectation or that's the assumption or something like that [...] I mean in a way it's a guilty feeling.

The expectation that sex 'will happen' results in Kaitlyn feeling guilty that it won't (Kaitlyn indicates she is not sex repulsed but does not want or desire sex). Here, we see the power of the norm - the expectation that sex will happen and that she will 'have sex' - and the guilt associated with refusing/ transgressing the norm. Kaitlyn takes up/embodies the construction of women as being responsible for men's sexual interest and satisfaction - so much so that the idea of not fulfilling this expectation is difficult for her to consider, that is, although asexual she feels as though she would eventually have sex. As an asexual identified woman who 'wants a partner and not have sex with them' Kaitlyn is thus constituted by, and constitutes herself through, these more prevalent discourses, even as she tries to challenge them. As Judith Butler says, 'discourses do actually live in bodies. They lodge in bodies; bodies in fact carry discourses as part of their own lifeblood' (Meijer and Prins, 1998: 282). This 
highlights the complex and often contradictory gendered discourses which inform responses of others to the possibility of an asexual romantic partner and the experiences and negotiations of participants as they engage in dating practices. However, the outcome for many of the participants has been the same - giving up on the 'dating game' for the time being.

\section{'Finding somebody' in BDSM communities}

While the majority of participants were not dating at the time of the interviews and many had given up on dating/finding somebody, Sarah was actively seeking a monogamous relationship. She had used both sexual and asexual websites with relatively little success. More recently Sarah had found that BDSM (Bondage and Discipline, Domination and Submission, and Sadism and Masochism) websites provided her with a number of intimate encounters and relationships. Although most of the literature produced by sexuality scholars or BDSM activists attributes BDSM's empowerment and pleasure to its nature as a sexual practice, Lorca Sloane, one of the first to research asexual encounters in BDSM spaces, argues that BDSM 'provides discursive spaces and conceptual frameworks for fostering and validating intimate exchanges that do not derive from or rely on sexual desire' $(2015$ : 552, 561). Sarah has experienced many intimate, non-sexual encounters through BDSM sites which she first discovered through a chance encounter:

Sarah (47): It wasn't my idea but somebody contacted me on a vanilla site and said, 'would you like a slave or something' [laughs], that might not have been the term but I thought, wow that sounded like a good idea. I felt very safe with him, you know, I felt like I wasn't going to get hassled, they just want to be told what to do. [...] So after I met him I did go onto a specific BDSM site. I've certainly met some nice people there.

On this BDSM site she does not identify as asexual but her profile indicates, 'there will be no sex'. While Sarah posted her asexual status on other dating websites, for BDSM sites she indicates that, 'they don't need to know why [I'm there], just these are my rules'. This was also the case for some of the asexual participants in Sloan's study who did not declare themselves as asexual but rather set the limits on what would happen and what constituted 'the no-touch areas' (2015: 556). The BDSM community is appealing to asexuals because activities are pre-negotiated, the non-genital focus of many BDSM practices, and the fact that some BDSM practitioners separate BDSM and genital sex (Bauer, 2010: 148). Thus, BDSM creates space for using activities that are conventionally equated with eroticism to create intimacies that they experienced as non-sexual (Sloan, 2015: 551). Sarah appreciates having control over what she will do and what will be done to her, as well as finding a space that has provided her with opportunities for non-sexual touch and intimacies. At the time of the interviews she had been 'seeing somebody' she met on a BDSM site for several years. 
There were three issues that Sarah raised in relation to her BDSM practices: what constitutes sex/a sexual action; how to define herself; and the non-monogamous norm in many BSDM communities (see Bauer, 2010). With respect to the first, saying that 'there will be no sex' does not necessarily ensure that a partner will not engage in an action that is sexual and unwanted. Sarah found that she had to be more specific with respect to saying what she wants as 'a lot of submissive men are really keen on going down on women [laughs], it makes them feel like they're serving, so I added that'. Nonetheless, she still encountered the odd person who 'broke the rules':

Sarah (47): I sent him the list before we met, and I don't really expect him to improvise, and he touched my vagina and I think he put his finger inside me and that seems like quite normal if you're vanilla, but to me that's not normal in that context. He's supposed to do what I want and if I wanted that to happen that would be on my list.

Sloan frames this as a conundrum faced by asexual individuals who seek relationships in BDSM spaces: 'declaring intercourse off-limits does not always provide adequate assurance that their partner does not hope for or expect them to desire or have sex, and they cannot always predict what behaviours would incite these expectations' (2015: 554). The second issue or conundrum is that Sarah encountered how to describe or define herself:

Sarah (47): Even on the BDSM site I have to admit that I'm not actually dominant. I had to tick the dominant box because otherwise l'd be a slave or something else [laughs], you have to pick one. But you know I had a lovely time with someone I met on there. But he needed to be humiliated and abused or something - that I could not manage. I mean I was crazy about him, he was lovely. I can be happy with them but they might not be happy with me. So they need to be a bit more borderline.

The requirement that one identifies as either dominant or submissive is problematic for Sarah who identifies as neither. Although she ticks 'the dominant box' this has put her in situations where she was unable to do what was expected of her. However, while she was happy with a 'submissive' partner, he was not happy with her. Sarah's ambivalent position with respect to BDSM categories means that she has to be clear about her position (and what she will/will not do) and engage with those she describes as more 'borderline'. Finally, Sarah was seeking a monogamous relationship in a community where non-monogamy is often the norm. She has been seeing a non-monogamous man for several years even though her stated aim is to find a monogamous relationship: 
Sarah (47): My aim is to [...] find somebody suitable.[...] For the last [few] years I've been seeing somebody who I met on a BDSM site, but he doesn't want to be monogamous, so he [...] is better than nothing, while I keep looking.

Sarah is committed to finding a monogamous relationship even though this search involves non-monogamous practices. Monogamy is thus framed as an ideal, future situation with 'someone suitable' where she will have no desire for intimate, non-sexual physical contact with another person:

Sarah (47): I feel like if I have the right person I will be [monogamous]. You know, I wouldn't want somebody else if there was someone suitable.

While the non-monogamous nature of many BDSM communities complicates searching for monogamy in these spaces, this does not deter Sarah. Like the majority of romantic-identified participants in my study, and those in Kristin Scherrer's (2010), monogamy often figures centrally. On the one hand, this reinscribes what Robin Bauer calls 'mono-normativity' which is 'based on the taken for granted allegation that monogamy and couple-shared arranged relationships are the principle of social relations per se' (2010: 145). On the other hand, it challenges the assumption that monogamy is shorthand for sexual monogamy and enables us to think about what monogamy might look like outside of sexual monogamy (Scherrer, 2010). For Sarah and a number of other participants, an ideal monogamous relationship involved physical intimacy (although this varied from holding hands to kissing and hugging), emotional intimacy, intellectual connection, shared interests, acceptance and respect.

\section{Conclusion}

This article has explored one aspect in the lives of primarily romantic identified asexuals living in New Zealand - their participation in the dating game (with non-asexuals) and attempts to find a partner. It is important to indicate that there are similarities between the experiences discussed in this paper and those many non-asexuals encounter when dating, for example, pressure to engage in sexual activity in an increasingly hook up culture and some women feeling guilty about 'leading on' male partners. However, for the romantic identified asexual participants in this study the crucial difference is the desire for a non-sexual relationship ('there will be no sex here'). Given the difficulties of meeting other asexuals (described as ideal partners), the participants used the same dating strategies as those around them, for example, connecting with potential dates in social situations (parties, bars, night clubs etc.) and online dating sites. The lack of awareness and understanding about asexuality they encountered (which often include abusive and derogatory comments) lead to participants negotiating when best to disclose their asexuality to a potential or actual date. However, in spite of different approaches, the result was usually the same - the termination of the date. With the exception of Sarah, who was hoping to find a relationship with someone in the BDSM 
community, all the participants had given up on dating and 'finding somebody' even though they still desired a partnered relationship.

A non-sexual partnered relationship (particularly between an asexual and non-sexual) is almost unintelligible in our increasingly sexualised, heteronormative culture, as highlighted by the responses participants received from potential dates (as well as family and friends in some cases). However, as suggested in this article, there is a gendered dimension to this which has received little attention in asexuality research to date. With respect to femininity, the legitimacy of the asexual female subject was denied through readings of female participants' lack of sexual interest or 'passivity' as simply in need of being awakened by a male partner. Participants responded to this is a number of ways. Some participants asserted the legitimacy of the female romantic asexual subject as one who needs no sexual awakening and who 'wants romance', refusing to position this as a precursor to sex. For example, Sarah's dating profile page said 'Asexual ahoy, touch yes: sex no' and she was happy to talk to and 'educate' those that contacted her. Other female participants also indicated that disclosure often involved explanation and discussion (education) about asexuality. However, at the same time, Madison and Kaitlyn articulate their guilt at 'leading on' male partners - so much so that Kaitlyn's guilt would potentially lead her to having sex. In other words, the discursive positioning of women as responsible for men's sexual satisfaction, once having aroused it, has been embodied by Kaitlyn such that it outweighs her sexual repulsion and ability to assert her asexual identity.

In a similar way the asexual male subject was unintelligible but for different reasons - you cannot be a guy/man and not be interested in sex. This is a difficult subject position for men who identify as aromatic asexual (as indicated by Philip), but for romantic identified asexual men who seek non-sexual, romantic, partnered relationships in which physical, non-sexual intimacy is desired (for example, Mike and Aidan) the responses of female dates was that male romantic asexuality could not be a 'real thing'. The result for Mike and Aidan was being dumped, being cheated on or dumping dates because they 'wanted more'. Although both men have given up on dating for the time being they acknowledge the ways in which romantic asexual masculinity contributes to reshaping and diversifying masculinities. Aidan for example, argues that although his 'asexuality is taking away things' it also 'stops me from being that hegemonic masculine [man]'. Although there has been recent research attention to changing forms of heteromasculinity (for example, see Holmes, 2015, on heterosexual men's capacity for embodied and relational forms of emotional reflexivity, and Anderson and McCormack's, 2015, examination of 'cuddling and spooning' practices among heterosexual young British men), the transgressive possibilities opened up by male romantic asexuality in which sex is detached from masculinity and romantic and emotional relationality are emphasised, have yet to be researched. In fact, as highlighted in this paper, the intersections of asexuality, gender and age are significant and in urgent need of further research by asexuality, feminist, critical sexualities and queer scholars. 


\section{Acknowledgements}

I am immensely grateful to the participants who gave their time to participate in this study.

\section{Notes}

\footnotetext{
${ }^{1}$ Māori are the indigenous people of New Zealand; Pakeha is the indigenous term for non-Māori.

${ }^{2}$ Approximately one third of participants had never met another self-identified asexual in person.

${ }^{3}$ It is difficult to know the number of asexuals, however, Bogaert (2004) estimates it to be about $1 \%$ of the population.

${ }^{4}$ For many of participants, identifying as asexual was framed as a 'private matter' which they haven't disclosed to many others. Mike, Aidan and Kaitlyn, for example, said 'it isn't anyone else's business'. ${ }^{5}$ Although the construction of middle and later life as a period of sexual decline has recently been challenged by a cultural scientific shift in which lifelong sexual function has become a primary component of achieving successful ageing (see Katz and Marshall, 2003), the framing continues to co-exist alongside the more recent 'sexy oldie' (Gott, 2005; Vares, 2009).

${ }^{6}$ This highlights the 'newer' construction of the sexually agentic female subject.
}

\section{Bibliography}

Anderson E and McCormack M (2015) Cuddling and spooning: Heteromasculinity and homosocial tactility among student-athletes. Men and Masculinities 18(2): 214-230.

Bauer R (2010) Non-monogamy in queer BDSM communities: Putting the sex back into alternative relationship practices. In: Barker $\mathrm{M}$ and D. Langdridge $\mathrm{D}$ (eds) Understanding Non-monogamies. London and New York: Routledge, pp. 142-153.

Bogaert A (2004) Asexuality: Prevalence and associated factors in a national probability sample. Journal of Sex Research 41(3): 279-287.

Bogle K (2007) The shift from dating to hooking up: What scholars have missed. Sociology Compass 1(2): 775-788.

Bogle K (2008) Hooking Up. New York: New York University Press.

Braun V and Clarke V (2006) Using Thematic Analysis in Psychology. Qualitative Research in Psychology 3:77-101.

Carrigan M (2011) There's more to life than sex? Difference and commonality within the asexual community. Sexualities 14(4): 462-478. 
Cerankowski K and Milks M (2014) Asexualities: Feminist and Queer Perspectives. Florence: Taylor and Francis.

Dawson M, McDonnell, L and Scott, S (2016) Negotiating the boundaries of intimacy: The personal lives of asexual people. The Sociological Review 64: 349-365.

Flore J (2014) Mismeasures of asexual desires. In: Cerankowski K and Milks M (eds) Asexualities: Feminist and Queer Perspectives. Florence: Taylor and Francis, pp. 52-86.

Foucault M (1990) [1978] The History of Sexuality: An Introduction. London: Penguin Books.

Garcia J, Reiber C, Massey S and Merriwether A (2012) Sexual hookup culture: A review. Review of General Psychology 16(2): 161-176.

Gavey N (1989) Feminist poststructuralism and discourse analysis. Psychology of Women Quarterly 13: 459- 475.

Gavey N (2005) Just Sex? The Cultural Scaffolding of Rape. London and New York: Routledge.

Gott M (2005) Sexuality, Sexual Health and Ageing. Berkshire, UK: Open University Press.

Gray J (1995) Mars and Venus in the Bedroom: A Guide to Lasting Romance and Passion. Rydalmere, NSW: Hodder and Stoughton.

Haefner C (2011) Asexual scripts: A grounded theory inquiry into the intrapsychic scripts asexuals use to negotiate romantic relationships. Ann Arbor, Michigan: UMI Dissertation Publishing, ProQuest.

Holmes M (2015) Men's emotions: Heteromasculinity, emotional reflexivity and intimate relationships. Men and Masculinities 18(2): 176-192.

Hollway W (1984) Gender difference and the production of subjectivity. In: Henriques J, Hollway W, Urwin C and Walkerdine V (eds) Changing the Subject: Psychology, Social Regulation and Subjectivity. London: Routledge, pp. 227-263.

Hollway W (1989) Subject and Method in Psychology: Gender, Meaning and Science. London: Sage.

Katz S and Marshall B (2003) New sex for old: Lifestyle, consumerism and the ethics of aging well. Journal of Aging Studies 17(1): 3-16. 
MacNeela P and Murphy A (2015) Freedom, invisibility, and community: A qualitative Study of self-identification with asexuality. Archives of Sexual Behavior 44: 700-812.

Meijer I and Prins B (1998) How bodies come to matter: An interview with Judith Butler. Signs: Journal of Women in Culture and Society 23: 275-286.

Plummer K (2005) Male sexuality. In: Connell R, Hearn J and Kimmel M (eds) The Handbook of Masculinity Studies. London and New York: Sage, pp. 178-195.

Przybylo E (2011) Crisis and safety: The asexual in sexusociety. Sexualities 14(4): 444-461.

Przybylo E (2013) Afterword: Some thoughts on asexuality as an interdisciplinary method. Psychology and Sexuality 4(2): 193-194.

Przyblo E and Cooper D (2014) Asexual resonances: Tracing a queerly asexual archive. GLQ: A Journal of Lesbian and Gay Studies 20(3): 297-318.

Robbins N, Low GF and Query A (2016) A qualitative exploration of the 'coming out' process for asexual individuals. Archives of Sexual Behavior 45: 751-760.

Scherrer K (2008) Coming to an asexual identity: Negotiating identity, negotiating desires. Sexualities 11(5): 621-641.

Scherrer K (2010) Asexual relationships: What does asexuality have to do with polyamory? In: Barker $\mathrm{M}$ and Landridge D (eds) Understanding Non-monogamies. London and New York: Routledge, pp. 154-159.

Scott S and Dawson M (2015) Rethinking asexuality: A symbolic interactionist account. Sexualities 18(1/2): 3-19.

Sloan L (2015) Ace of (BDSM) clubs: Building asexual relationships through BDSM practice. Sexualities 18(5/6): 548-563.

Van Houdenhove E, Gis L, T'Sjoen G and Enzlin P (2015) Stories about asexuality: A qualitative study on asexual women. Journal of Marital and Sex Therapy 41(3): 262-281.

Vares T (2009) Reading the 'sexy oldie': Gender, age(ing) and embodiment. Sexualities 12(4): 503-524.

Weedon C (1987) Feminist and Poststructuralist Theory. Oxford, UK: Basil Blackwell. 